\title{
Grado de implementación del proceso de seguridad en el paciente en IPS odontológicas públicas y privadas en Santa Marta*
}

\section{Implementation Degree of the Patient Safety Process in Public and Private Dental IPS in Santa Marta}

\section{Grado de implementação do processo de segurança no paciente em IPS odontológicas públicas e privadas em Santa Marta}

Fecha de recepción: 20-10-2016 Fecha de aceptación: 15-12-2016 Disponible en línea: 30-05-2017

Cómo citar este artículo:

doi:10.11144/Javeriana.rgps16-33.gips

Vila-Sierra LA, Salcedo-Salgado JD, Fernández-Roncallo YY, Narváez-Barandica MM. Grado de implementación del proceso de seguridad en el paciente en IPS odontológicas públicas y privadas en Santa Marta. Rev Gerenc Polít Salud. 2017; 16 (33): 116-125. https://doi.org/10.11144/Javeriana.rgps16-33.gips

\author{
Luis Armando Vila-Sierra** \\ Juan David Salcedo-Salgado** \\ Yelaine Yamile Fernández-Roncallo*** \\ Mayuri Milena Narváez-Barandica****
}

\footnotetext{
* $\quad$ Artículo de investigación realizado por estudiantes y docentes de la Universidad del Magdalena, de la ciudad de Santa Marta, resultado del trabajo de grado titulado Grado de implementación del proceso de seguridad en el paciente en IPS odontológicas públicas y privadas en Santa Marta, para optar al título de odontólogos.

** Candidato a doctor, magíster en Desarrollos Sociales-Salud Familiar, Universidad del Norte, Colombia; magíster en Ciencias Sociales y Humanas, Universidad de París; magíster en Gerencia en Salud y en Auditoría de la Calidad en los Servicios de Salud, Universidad Jorge Tadeo Lozano; especialista en Docencia Universitaria, Universidad El Bosque; docente de la Universidad del Magdalena. Correo electrónico: luvila25@yahoo.com

*** Especialista en Gerencia y Auditoria de la Calidad, Universidad Jorge Tadeo lozano; especialista en Docencia Universitaria, Universidad E1 Bosque; catedrático de la Universidad del Magdalena. Correo electrónico: juandsal-

$116 * * * * \begin{aligned} & \text { cedo@gmail.com } \\ & \text { Estudiante de grado, Universidad del Magdalena. Correo electrónico: yelaine2014@gmail.com }\end{aligned}$

***** Estudiante de grado, Universidad del Magdalena. Correo electrónico: siruyram270@gmail.com
} 


\title{
Resumen
}

El propósito de esta investigación es determinar el grado de implementación del proceso de seguridad en el paciente en IPS odontológicas de Santa Marta. Es un estudio descriptivo, cuya muestra está conformada por doce instituciones, siete públicas y cinco privadas. Se aplicó una encuesta analizada con el programa estadístico SPSS 20.0. Todas las entidades privadas cuentan con un programa de seguridad del paciente, mientras que solo tres de las siete entidades públicas pueden validarlo. El $58 \%$ de las instituciones muestran un programa de capacitaciones. La política de seguridad se pudo validar en un $58 \%$ de las IPS, mientras que para los criterios de validación de procesos, procedimientos y formatos solo ocho de las doce entidades lo evidencian y dos de estas instituciones solo reportaron registros. Se evidencia que la causa más frecuente de eventos adversos en las IPS públicas es atribuible a fallas administrativas y de adherencia, mientras en las IPS privadas prevalecen las causas atribuibles a los procedimientos.

Palabras clave: eventos adversos; odontología; seguridad del paciente

\begin{abstract}
The purpose of this research is to determine the implementation degree of the patient safety process in Santa Marta dental IPS. This is a descriptive study, whose sample is made up of twelve institutions, seven public and five private. A survey was carried out using the statistical program SPSS 20.0. All private entities have a patient safety program, while only three out of the seven public entities could prove to have it. $58 \%$ of the institutions show a training program. The security policy could be validated in $58 \%$ of the IPS, whereas for the validation criteria of processes, procedures, and formats only eight of the twelve entities met them, and two of these institutions only reported records. It could be seen that the most frequent cause of adverse events in public IPS institutions is attributable to administrative failures and adherence, whereas in private IPS institutions there is a prevalence of causes attributable to the procedures.
\end{abstract}

Keywords: adverse events; odontology; patient safety

\section{Resumo}

O propósito desta pesquisa é determinar o grau de implementação do processo de segurança no paciente em IPS odontológicas de Santa Marta. Trata-se de um estudo descritivo, cuja amostra está conformada por doze instituições, sete públicas e cinco privadas. Aplicou-se inquérito analisado com o programa estatístico SPSS 20.0. Todas as entidades privadas contam com um programa de segurança do paciente, enquanto apenas três das sete entidades públicas podem validá-lo. $58 \%$ das instituições mostram um programa de capacitações. A política de segurança pôde validar $58 \%$ das IPS, enquanto para os critérios de validação de processos, procedimentos e formulários apenas oito das doze entidades evidenciam-no e duas destas instituições apenas reportaram registros. Evidencia-se que a causa mais frequente de eventos adversos nas IPS públicas é atribuível a falhas administrativas e de aderência, enquanto nas IPS privadas prevalecem as causas atribuíveis a procedimentos.

Palavras-chave: eventos adversos; odontologia; segurançae 


\section{Introducción}

La seguridad del paciente ha tenido gran relevancia porque se enfoca en mejorar la seguridad de la atención y prevenir posibles afectaciones en los pacientes (1) lo cual la convierte en un lineamiento fundamental que influye en la calidad de la atención. Se realizaron publicaciones aisladas con respecto a la seguridad del paciente en odontología, pero ninguna de ellas fue tema de discusión entre los miembros de la comunidad científica. Dichas publicaciones solo transcendieron en 1999, en el documento Errar es humano, del Instituto de Medicina de los Estados Unidos (2), donde se reporta que en los Estados Unidos de América, alrededor de cien mil pacientes morían cada año, no como producto de sus enfermedades, sino por errores en la atención médica (3).

En tal sentido, la Organización Mundial de la Salud calcula que en los países desarrollados uno de cada diez pacientes sufre algún tipo de daño durante su estancia hospitalaria, y en países en desarrollo esta cifra se eleva al $25 \%$ de las atenciones. Entre tales daños se encuentran las infecciones (4-6). Específicamente en Colombia, trece de cada cien pacientes sufren un evento adverso que puede causarles la muerte, y las principales causas se asocian a las infecciones intrahospitalarias y las deficiencias asociadas al uso de medicamentos (7). Recientemente, se reportó validación de seguimiento a eventos adversos odontológicos donde se priorizan los ocurridos en el servicio endodóntico (8), lo cual constituye un problema grave de salud pública.

Con el fin de controlar los eventos adversos en la atención en salud, en el año 2007 Colombia suscribió la Alianza Mundial para la Seguridad del Paciente, creada por la Organización Mundial de la Salud, la cual busca estandarizar prácticas que brinden mayor seguridad en los hospitales y en los sistemas de salud $(5,9)$. Es así como los prestadores de servicios de salud han basado la gestión del riesgo en un enfoque epidemiológico que busca minimizar la probabilidad de eventos prevenibles, trauma o muerte que afectan al individuo (10) y asi brindar una mejor seguridad al paciente (5).

De ahí la importancia de reflexionar sobre la magnitud que tiene el problema de los eventos adversos en salud oral, para prevenir su ocurrencia y progresar en acciones que garanticen una práctica segura (11).

Por esta razón, al observar las cifras de incidencia con respecto a los servicios de salud en Colombia, luego de llevarse a cabo una mesa de trabajo donde participaron representantes de IPS públicas (12), se presentó un incremento del $43 \%$ en el reporte de indicadores del 2009, a pesar de lo cual el registro de estos sucesos sigue encontrándose en un nivel bajo, de acuerdo a lo referenciado por la OMS $(4,5)$.

Por lo tanto, es necesario que como parte del seguimiento del Estado, todas las IPS reporten mensualmente, de forma obligatoria, indicadores de calidad tales como: seguridad en la administración de medicamentos, seguridad en la identificación de procedimientos para los pacientes, reducción de perforaciones endodónticas, etcétera, para controlar los eventos adversos (13). Por esto, el Ministerio de Salud y Protección Social publicará y mantendrá actualizado el catálogo y las fichas de los indicadores trazadores y las estimaciones de los indicadores disponibles a partir del reporte del año en curso. Así, se conformará una fuente de información para las entidades del Sistema General de Seguridad Social en Salud (SGSSS) y los organismos de inspección, vigilancia y control, en lo que corresponde al monitoreo de la calidad en salud (14).

En la actualidad sanitaria odontológica, el objetivo fundamental de la "seguridad del 
paciente” es evitar, en la medida de lo posible, los sucesos adversos evitables (inccidentes o errores) asociados a la asistencia, para impedir que vuelvan a suceder (15). En la consulta odontológica el paciente también está expuesto a sufrir eventos adversos derivados de las actuaciones clínicas $(9,16)$, los cuales ocurren por diferentes causas (17), aunque a escala hospitalaria se estima que el mayor número de eventos adversos en odontología (hasta un $50 \%$ ) se registra en el uso de fármacos (14). Sin embargo, la asistencia odontológica ambulatoria presenta menor impacto que la asistencia hospitalaria y, consecuentemente, genera daños más leves $(15,18,19)$.

Dado que la seguridad del paciente es una prioridad en la gestión de calidad, el propósito de esta investigación fue constatar si las IPS odontológicas de Santa Marta desarrollan un programa de seguridad del paciente que garantice una atención oportuna, conforme a los estanderes de calidad pertinente, accesible y continua, y que disminuyan los eventos adversos, con lo cual mejora la seguridad del paciente. De otra parte, se buscó determinar cuáles son las causas más frecuentes de sucesos en atención odontológica.

\section{Materiales y metodos}

Esta investigación, de corte transversal descriptiva, fue realizada por los estudiantes Bernardo Arnedo, Lauren Díaz y María Acosta y contó con la asesoría de los doctores Luis Vila y Juan Salcedo. El estudio fue realizado con doce IPS, siete públicas y cinco privadas, lo cual correspondió al $17 \%$ del total de las instituciones que cumplieran con los criterios y estuviesen registradas en Santa Marta. La selección se realizó con un criterio de muestreo intencional o de conveniencia, ya que las entidades escogidas fueron aquellas que presentaron la disposición y disponibilidad para el desarrollo de la investigación. Como criterio de inclusión se estableció, aimismo, que fueran prestadores con servicios en salud oral que estuviesen inscritos en el Registro Especial de Prestadores (REPS) ofertando servicios para Santa Marta. Se excluyeron los profesionales independientes, para minimizar sesgos de informacion, en razón al bajo volumen de atenciones de estos profesionales.

A cada una de las entidades se le aplicó una encuesta, previamente validada, en la cual se realizaron una serie de preguntas de tipo cerrado donde solo se podía afirmar o negar, por medio de una respuesta concreta sobre lo que se preguntaba. También hubo algunas preguntas abiertas, en las cuales el entrevistado debía identificar y establecer un concepto sobre lo indagado. Por otra parte, el análisis estadístico de los resultados obtenidos se realizó con el programa estadístico SPSS Inc. 20.0, donde se ingresaron los datos concernientes a los valores que arrojó la encuesta de las diferentes variables aplicadas a las entidades, con relación a la problemática tratada en la investigación.

Para establecer las causas odontológicas más frecuentes de eventos adversos se evaluaron los servicios habilitados por las IPS, las cuales se identificaron según su naturaleza pública o privada. Estas debían cumplir con el sistema unico de habilitación, indispensable para la entrada y permanencia en el Sistema. Así, se busca dar seguridad a los usuarios frente a los riesgos potenciales asociados a la prestación de servicios odontológicos. Los requisites de habilitacion son de obligatorio cumplimiento para toda oferta de servicios de salud (18-21).

También se analizó el Programa de Seguridad del Paciente y su desempeño, mediante el cumplimiento de categorías definidas, orientadas a la seguridad de cada una de los servicios, tomando en cuenta elementos tales como conceptualización, documentación, socialización, implenetación y seguimiento del programa en 
mención, con el fin de prevenir la ocurrencia de eventos adversos y contar con instituciones seguras y competitivas en el contexto internacional (21-23).

Para el concepto de validacion del Programa se debían evidenciar registros que cumplieran con los siguientes criterios: politica de seguridad, procesos, procedimientos, formato y registros.

De esta forma, se evidenciaron las causas de mayor frecuencia para la materialización de eventos adversos ocasionados por el incumplimiento con el Programa, los cuales son resultado de errores durante la atención en salud que, de manera no intencional, producen daño (10) y pueden ser prevenibles (resultado no intencional que se pudo haber prevenido aplicando todos los estándares de cuidado asistenciales) o no prevenibles $(8,24,25)$, resultado no intencional que se presenta a pesar de haber cumplido todos los estándares relacionados con los cuidados asistenciales $(26,27)$. Por esto, las instituciones deben definir un protocolo para el manejo del paciente y su familia cuando ocurre un evento adverso (28-30).

Se determinó que la implementación del proceso de seguridad en clínicas odontológicas de Santa Marta se validaría por medio del cumplimiento con los criterios sobre el desarrollo del Programa de Seguridad del Paciente en cada una de las entidades.

\section{Declaraciones de aspectos éticos}

La investigcación se realizó siguiendo los principios de investigación éticos de acuerdo con los principios de Helsinki (Modificación de Edimburgo 2000), contemplados en la normatividad colombiana por la Resolución 8430 de 1993 del Ministerio de Salud, "Por la cual se establecen las normas científicas, técnicas y administrativas para la investigación en salud". De esta manera, se firmó el proceso de consentimiento informado y cada entidad suministró la información solicitada por medio del instrumento. De igual forma, se protegió la privacidad de cada una de las entidades participantes, mediante acuerdo de confidencialidad.

\section{Resultados}

Se incluyeron doce entidades, de las cuales siete son estatales o de carácter público y cinco son privadas. Con respecto a la disponibilidad del Programa de Seguridad del Paciente, se encontró que del total de las instituciones estudiadas, solo el $66 \%$ contó con este (véase figura 1).

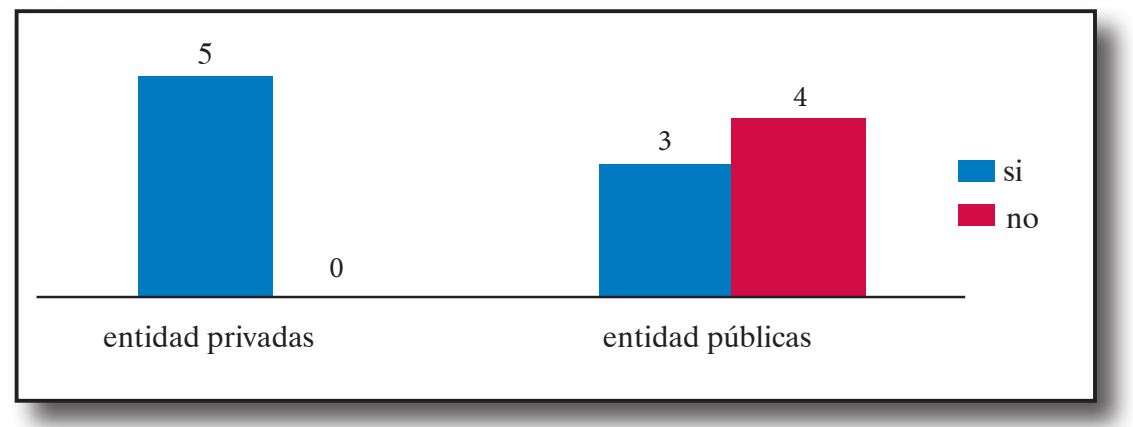

FIGURA 1. ¿Se Cuenta con un Programa de Seguridad del Paciente en la ciudad de Santa Marta?

Fuente: elaboración propia 
En relacion con los criterios del Programa, de las doce entidades estudiadas en el criterio de conceptualización, el $42 \%$ validó este proceso. En la etapa de documentacion, igualmente el $42 \%$ de las entidades evidenció esta fase, mientras que en la etapa de socialización el $33 \%$ validó el cumplimiento; sin embargo, en las etapas de implementación, seguimiento y análisis ninguna de las instituciones entrevistadas mostró avances significativos (véase figura 2) .

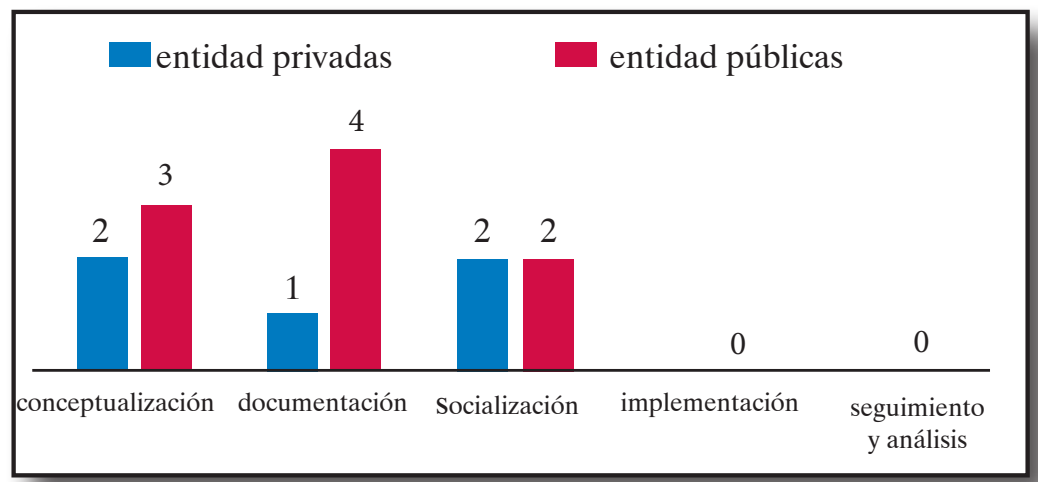

Figura 2. Programa de Seguridad del Paciente en la ciudad de Santa Marta, año 2014

Fuente: elaboración propia

E1 uso de capacitaciones sistemáticas mostró que el 58\% de las instituciones validó estas actividades, con un comportamiento semejante en el incumplimiento entre instituciones publicas y privadas (véase figura 3). De igual forma, en el criterio de inducciones, el $41 \%$ de las IPS realizó este procedimiento, pero el $58 \%$ no pudo validar este criterio (véase figura 4).

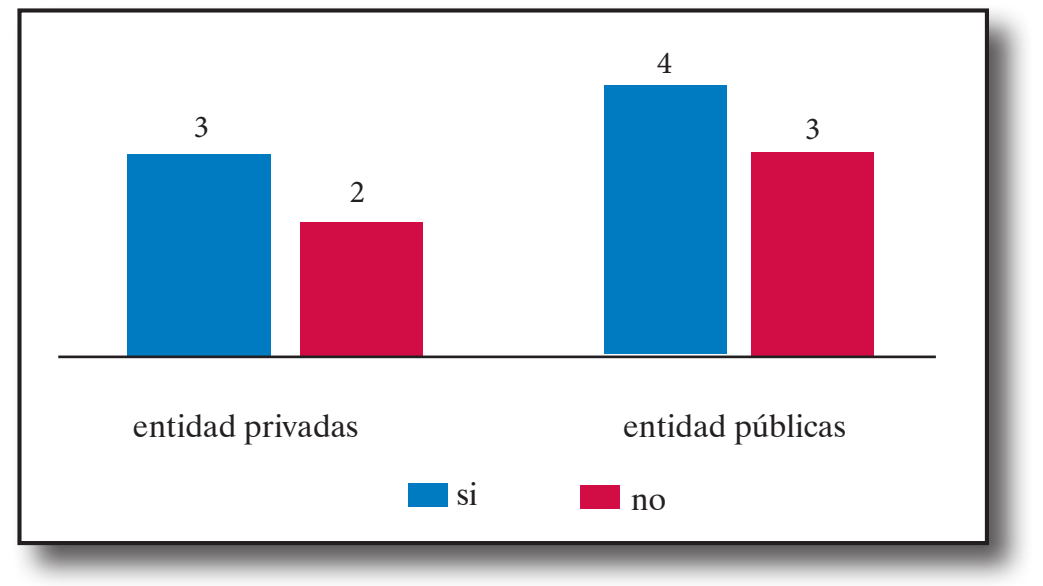

FIGURA 3. CUMPLIMIENTO DE LAS CAPACITACIONES SISTÉMICAS EN LAS IPS DE LA CIUDAD DE SANTA MARTA, AÑO 2014

Fuente: elaboración propia 


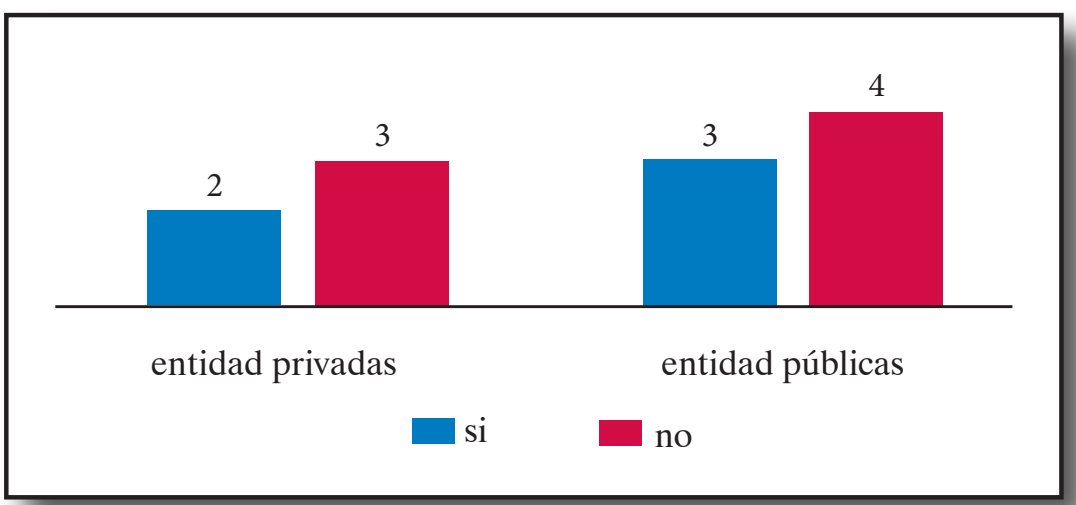

FigURA 4. Prestadores Que cumplen con inducciones en la ciudad de Santa Marta, aÑo 2014

Fuente: elaboración propia

En el proceso de validación del Programa se pudo observar que, en el ítem de política de seguridad, el $58 \%$ de las IPS presentaron cumplimiento con el enfoque, en tanto que en los ítems de validacion de procesos, procedimientos y formatos, ocho de las estas presentaron evidencia, y solo dos de las instituciones públicas presentaron reportes en la atención de registros (véase figura 5).

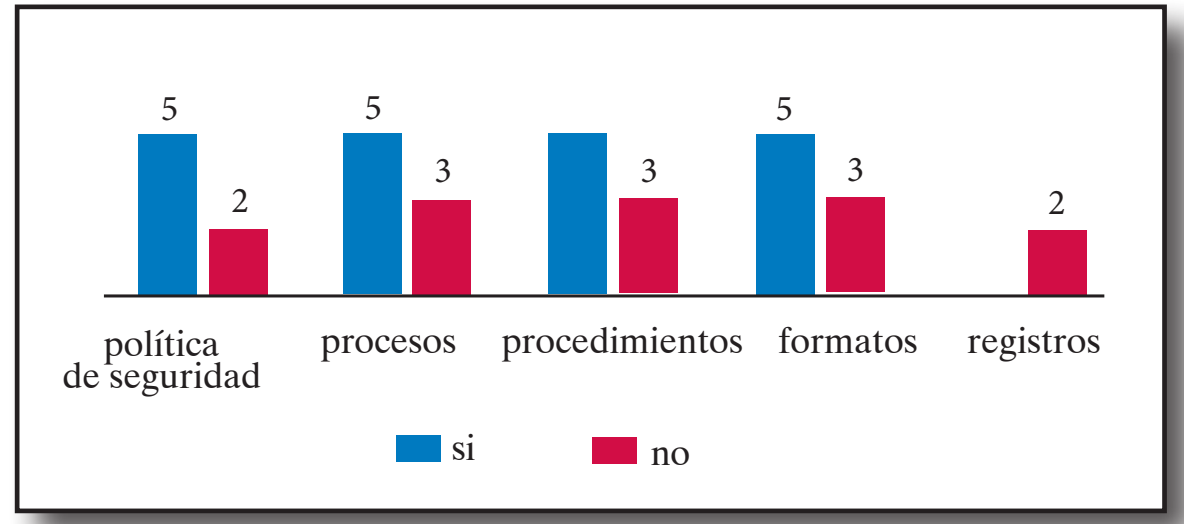

Figura 5. Criterios de validación en el Programa de Seguridad del Paciente en la ciudad de Santa MARTA, AÑo 2014

Fuente: elaboración propia

En cuanto a las causas de eventos adversos en los servicios odontológicos, se evidenció que en las instituciones públicas los eventos adversos de mayor prevalencia se asociaron con causas administrativas y la adherencia de procedimientos, mientras que en las IPS privadas predominaron la causas relativas a los procedimientos (véase figura 6). 


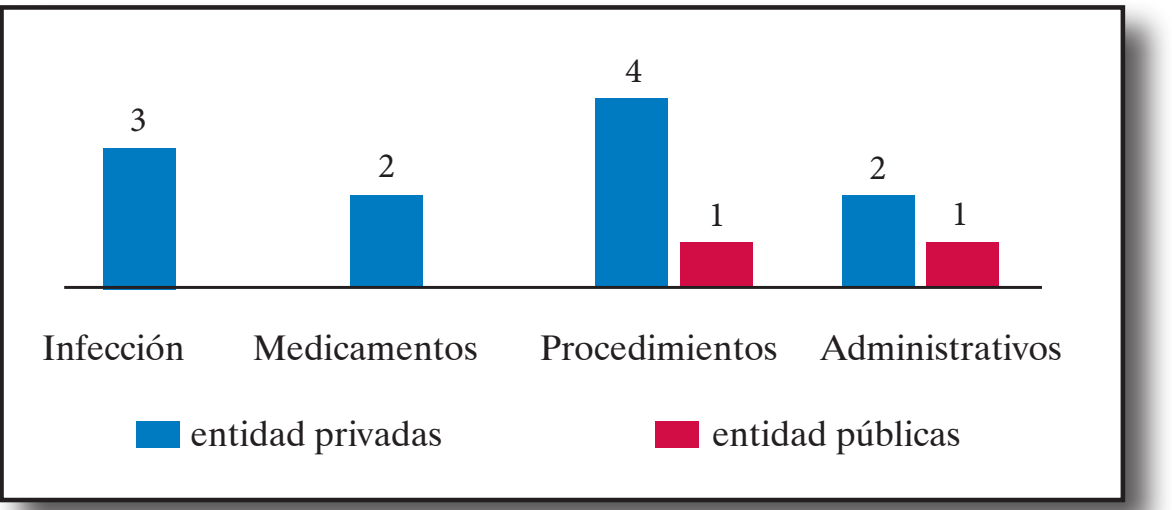

FigURA 6. Causas asociadas a los eVentos adVersos en la ciudad de Santa Marta, año 2014

Fuente: elaboración propia

\section{Discusión}

Salcedo y Vila (8) hace referencia a la distribución de los eventos adversos según su ocurrencia, con mayor impacto en el uso de biomateriales (alergias) y al manejo del paciente con enfermedades sistémicas. También menciona que la principal causa de eventos adversos es por procedimiento y, a diferencia de nuestra investigación, esta es la tercera causa más frecuente de incidentes, debido a fallas en el cumplimiento de los procedimientos definidos en protocolos de manejo.

En otro estudio, realizado por la OESPO (30), se muestra de forma explícita dónde son más frecuentes los eventos adversos de la consulta odontológica, y en primer lugar se encuentran los errores terapéuticos, a diferencia de los resultados obtenidos en el presente estudio, donde se hicieron frecuentes los eventos adversos por procedimientos.

Según la Organización Mundial de la Salud, para año 2014 la seguridad del paciente se había convertido en un problema grave para muchos países, tanto desarollados como en desarrollo, debido a que en la atención hospitalaria, mediante los procedimientos, por lo menos uno de cada diez pacientes sufren algun daño. Para nosotros, es de gran importacia conocer el grado de implementación de la seguridad del paciente en las IPS estudiadas de la ciudad de Santa Marta, ya que a través de ello se observa que la principal causa de eventos adversos se asocia con el momento de realizar procedimientos. Por esto, es necesario que las instituciones promuevan entre los profesionales de la salud el cumplimiento de las capacitaciones que se brindan para mejorar la atención al paciente, y evitar la pequeña frase errar es humano, según el Instituto de Medicina de los Estados Unidos.

\section{Conclusión}

La seguridad del paciente no deja de ser una problemática para muchas instituciones prestadoras del servicio odontológico en la realización de procedimientos. Aunque es común que esta clase de sucesos ocurra, estos suelen ser más leves que los que se presentan en los servicios intrahospitalarios. Es importante hacer seguimiento y gestión de los eventos adversos en las instituciones, así como implantar una cultura de seguridad del paciente, para poder mejorar los servicios sanitarios odontológicos 


\section{LUIS ARMANDO VILA-SIERRA • JUAN DAVID SALCEDO-SALGADO • \\ Yelaine YAMILE FERnÁNDEZ-RonCALlo • MAYURI MILENA NARVÁEZ-BARANDICA}

Si bien en ninguna de las instituciones que participaron del estudio se validó el cumplimiento total de los criterios definidos para la seguridad del paciente, en algunas instituciones privadas se evidencia un mayor cumplimiento de los criterios definidos, con un $64 \%$. Colombia ha hecho su máximo esfuerzo para mejorar los estándares y el seguimiento de la seguridad de los pacientes. Como muestra, en el 2005 el Sistema Único de Acreditación en Salud introdujo como requisito de entrada a las instituciones que quisieran acreditarse, la exigencia de tener un sistema de reporte de eventos adversos.

Hoy nuestro país cuenta con una Política Nacional de Seguridad del Paciente y una Guía Técnica de Buenas Prácticas. Para una mejor atención odontológica, se elaboró un paquete educativo que instruye al lector sobre cómo implementar la práctica en su lugar de trabajo y evaluar su impacto. De tal manera, con este estudio es posible evidenciar avances en el cumplimiento de la normatividad congruentes con el principio de la secuencialidad que implica la cultura de la IPS segura. Por esto, las instituciones deben vigilar periódicamente (auditorías internas) que el profesional de la salud siga las guías de práctica clínica y los protocolos de seguridad del paciente. Es recomendable que las IPS conozcan sobre el proceso de implantación de las guías de práctica clínica, así como sobre el grado de adherencia en el equipo odontológico y, de esta manera, identificar las fallas al momento de realizar los procesos y procedimientos, evitando así posibles eventos adversos.

\section{Referencias bibliograficas}

1. Alianza Mundial para la Seguridad del Paciente. La investigación de seguridad del paciente. Ginebra: Organización Mundial de la Salud; 2008.

2. Archila C, Coelli M. la seguridad del paciente en la prestación del servicio de salud. Bucaramanga: Universidad Nacional de Santander; 2008.
3. Colombia, Ministerio de la Protección Social. Guía técnica "Buenas prácticas para la seguridad del paciente en la atención en salud”. Bogotá: Ministerio de la Protección Social; 2010.

4. Organización Mundial de la Salud. 10 datos sobre seguridad del paciente. 2014. Disponible en: http:// www.who.int/features/factfiles/patient_safety/es/

5. Silva D, López C. Eventos adversos en los pacientes ambulatorios de una IPS de primer nivel en la ciudad de Medellín en el periodo de junio a diciembre de 2009. Medellín: Universidad CES; 2010.

6. Facultad de Medicina del Salvador. Guía curricular sobre la seguridad del paciente. Versión preliminar en español. s .1.: OMS; 2012.

7. Coomeva. Seguridad del paciente. Cero fallas médicas y clínicas. Revista Salud [internet]. 2014 [acceso: 3 de noviembre de 2015]. Disponible en: http://www. coomeva.com.co/publicaciones.php? $\mathrm{id}=37656$

8. Salcedo J, Vila L. Revisión sistemática de eventos adversos en el servicio de odontología. Rev CIO [internet]. 2014 [acceso: 21 de julio de 2013]; 5(14):111. Disponible en: http://www.rcio.org/index.php/ rcio/article/view/180/321

9. Espina M, Yepes L, García F. Seguridad del paciente: aspectos generales y conceptos básicos. Medellín: Universidad CES; 2010.

10. Puerto N. La gestión del riesgo en salud en Colombia. Bogotá D. C.: Universidad Nacional de Colombia; 2011.

11. Mojica J. Manual de buenas prácticas para la atención y seguridad del paciente. Bogotá: Editorial Universidad Militar Nueva Granada; 2014.

12. Colombia, Ministerio de Salud y Protección Social. Boletín Salud [internet]. Disponible en: https:// www.minsalud.gov.co/.../BOLETIN \% 20SALUD. pdf

13. Colombia, Gobernación del Valle del Cauca. Estándares de habilitación en salud. Procesos prioritarios para IPS [internet]. 2014. Disponible en: http://www.valledelcauca.gov.co/salud/descargar. php?id=14064

14. Colombia, Ministerio de Salud y Protección Social. Resolución 256 de 2016 (internet). Disponible en https://www.minsalud.gov.co/.../Resolución \%20 0256\%20de \%202016.pdf

15. Africano L, Antolinez J, Uribe E. Diseño de un programa de vigilancia y reporte de eventos adversos centrado en la seguridad del paciente para la clínica metropolitana de Bucaramanga S. A. Bucaramanga: Universidad CES - Universidad Autónoma; 2010.

16. Dental. Número especial monográfico: Seguridad del paciente en odontología. Rev CFC [internet]. 2011 [acceso: 9 de noviembre de 2015]; 8(1):7-71. Disponible en: http://pendientedemigracion.ucm.es/ centros/cont/descargas/documento27208.pdf

17. Maldonado M. Prevención de la mala praxis. Buenos Aires: Editorial Gador; 2007.

18. Colombia, Ministerio de Salud y Protección Social. Resolución número 00002003 de 2014. Bogotá D. C.: Ministerio de Salud y Protección Social; 2014.

19. Actualización para odontología - Resolución 2003 de 2014. Farpag.com. Disponible en: http://www.farpag. com/pdf/Resolucion2003de2014.pdf 
20. Colombia, Ministerio de Salud. Guía técnica "Buenas prácticas para la seguridad del paciente en la atención en salud". Seguridad del paciente y la atención segura. Versión 2.0. Bogotá: Ministerio de Salud; 2010.

21. Polanco M. Programa de seguridad del paciente. Versión 1. Código GC-S4D4. s. 1.: ESE Carmen Emilia Ospina; 2013.

22. Luengas S. Seguridad del paciente: conceptos y análisis de eventos adversos. Vía SALUD [internet]. 2009 [acceso: 7 de noviembre de 2015]; (48):6-21. Disponible en: www.cgh.org.co/imagenes/calidad1.pdf

23. Gobierno de España. Plan de calidad para el sistema nacional de salud. Siete pasos para la seguridad del paciente en la atención primaria [internet]. Disponible en: http://www.msssi.gob.es/organizacion/sns/ planCalidadSNS/docs/siete_pasos_seguridad_pacieante.pdf

24. Colombia, Ministerio de la Protección Social. Lineamientos para la implementación de la política de seguridad del paciente. Bogotá D. C.: Ministerio de la Protección Social; 2008.

25. Teheran C, Correa E, Mazenett E. Perfil epidemiológico de Cartagena de Indias. Cartagena de Indias: Departamento Administrativo Distrital de Salud (DADIS); 2011.
26. Perea B. Seguridad del paciente y odontología. Rev Cient Dent [internet]. 2011 [acceso: 5 de noviembre de 2015]; 8(1):9-15. Disponible en: http://pendientedemigracion.ucm.es/centros/cont/descargas/ documento23390.pdf

27. Aguado F, Aguado J, Lillo C. Emergencias en odontología. Barcelona: Editorial Glosa; 2010.

28. Medrano E, Starlight C, Medrano J, Casas C. Asociación entre el motivo de consulta y el diagnóstico en pacientes de la Climuzac. Rev RIC [internet]. 2014 [acceso: 7 de noviembre de 2015]; 3(6):1-12. Disponible en: http://dialnet.unirioja.es/descarga/ articulo/5045741.pdf

29. Arce D, Oliveros M, Rivera J, Ruiz D. Programa de seguridad del paciente reporte y gestión de eventos adversos terapia respiratoria unidad de cuidado intensivo adulto Fundación Hospital San José Guadalajara de Buga. Manizales: Editorial Universidad Católica de Manizales; 2012.

30. Perea B. OESPO, nace para potenciar la cultura de la seguridad en odontología, Madrid- España 2012 disponible en: http://www.gacetadental.com/2012/04/ oespo-nace-para-potenciar-la-cultura-de-la-seguridad-en-odontologia-24585/ 